\title{
Cannabidiol increases gramicidin current in human embryonic kidney cells: An observational study
}

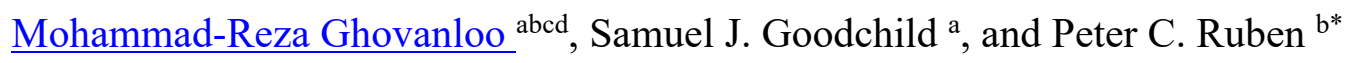

a) Department of Cellular and Molecular Biology, Xenon Pharmaceuticals, Burnaby, BC, Canada.

b) Department of Biomedical Physiology and Kinesiology, Simon Fraser University, Burnaby, BC, Canada.

Current affiliation:

c) Department of Neurology, Center for Neuroscience \& Regeneration Research, Yale University School of Medicine, New Haven, CT, USA.

d) Neuro-Rehabilitation Research Center, Veterans Affairs Connecticut Healthcare System, West Haven, CT, USA.

* Corresponding Author: Dr. Peter C. Ruben, Department of Biomedical Physiology and Kinesiology, Simon Fraser University, 8888 University Drive, Burnaby, BC, Canada V5A 1S6. e-mail: pruben@sfu.ca

phone: 778-782-6852

fax: $778-782-3040$ 


\begin{abstract}
Gramicidin is a monomeric protein that is thought to non-selectively conduct cationic currents and water. Linear gramicidin is considered an antibiotic. This function is considered to be mediated by the formation of pores within the lipid membrane, thereby killing bacterial cells. The main non-psychoactive active constituent of the cannabis plant, cannabidiol (CBD), has recently gained interest, and is proposed to possess various potential therapeutic properties, including being an antibiotic. We previously determined that CBD's activity on ion channels could be, in part, mediated by altering membrane biophysical properties, including elasticity. In this study, our goal was to determine the empirical effects of CBD on gramicidin currents in human embryonic kidney (HEK) cells, seeking to infer potential direct compound-protein interactions. Our results indicate that gramicidin, when applied to the extracellular HEK cell membrane, followed by CBD perfusion, increases the gramicidin current.
\end{abstract}

Keywords: cannabidiol, gramicidin, voltage-clamp, membrane 


\section{INTRODUCTION}

Linear gramicidins are a family of antibiotics whose function is determined by increasing the cationic permeability of the membrane (Harold \& Baarda, 1967; Hladky \& Haydon, 1970). Increased permeability is achieved by the formation of bilayer-spanning channels via dimerization of two hemi-channels. Relative to the channels formed by other antibiotics, gramicidin (gA) is well-behaved, and forms channels that are cation selective. Gramicidin channels are also among the best-understood of these types of channels. Atomic-resolution structures have been provided, and a wealth of functional experiments have yielded important insights into gA function (Anon, n.d.; Andersen \& Koeppe, 2007). Gramicidin channel monomers that reside in each membrane leaflet must dimerize with monomers in the other leaflet to form a continuous pore. This conformational change is necessary and sufficient for cationic currents to be conducted through gA. The pore diameter is $\sim 4 \AA$, sufficient to allow the pore to also conduct alkali metals, protons, and water (Hladky \& Haydon, 1970, 1972; Finkelstein, 1974; Andersen et al., 2005).

The rate of gramicidin channel dimerization is directly related to membrane stiffness or elasticity (Andersen \& Koeppe, 2007; Kapoor et al., 2019). This property has been the foundation of functional assays designed to determine the effects of various compounds on membrane dynamics. For example, compounds that reduce the membrane stiffness or thickness (e.g. detergents) enhance the probability of gramicidin dimerization, which in turn increases the cationic gramicidin signal (Lundbæk et al., 2004; Lundbæk, 2005; Ingólfsson et al., 2010; Kapoor et al., 2019).

Amphiphiles are among the compounds characterized using the gramicidin-based assays (Lundbæk, 2005). Amphiphilic compounds are a set of molecules possessing both lipophilic and hydrophilic properties. These molecules often display non-selective modulatory effects on seemingly unrelated targets, a by-product of amphiphiles modulating membrane elasticity (Lundbæk et al., 2004; Lundbæk, 2005; Kapoor et al., 2019). Modulation is achieved when amphiphiles localize at the solution-bilayer interface, which is made possible by the compounds' polar group residing at the interface with the hydrophobic region, which then inserts into the bilayer core. Partitioning into the lipid bilayer alters membrane elasticity, and changes phase preference and membrane curvature (Lundbæk et al., 2004; Lundbæk, 2005; Kapoor et al., 2019). 
One compound with amphiphilic properties is cannabidiol (CBD), the primary nonpsychotropic constituent of Cannabis sativa (Elsohly, 2007). CBD is a clinically and experimentally substantiated therapeutic compound with efficacy against a variety of conditions, including seizure disorders (for which CBD is FDA-approved), pain, and muscle spasms (Ross et al., 2008; De Petrocellis et al., 2011; Devinsky et al., 2017; Kaplan et al., 2017; Ghovanloo et al., 2018; Iannotti et al., 2019; Fouda et al., 2020). Furthermore, CBD has been suggested to have antibiotic properties (van Klingeren \& ten Ham, 1976; Kosgodage et al., 2019). Unlike the psychotropic $\Delta 9$-tetrahydracannabinol (THC), CBD has little to no affinity for endocannabinoid receptors (Devane et al., 1988; Lupica et al., 2004). However, many studies have shown that CBD interacts with a wide range of other targets, including a diverse array of ion channels (Ross et al., 2008; De Petrocellis et al., 2011; Kaplan et al., 2017; Ghovanloo et al., 2018, 2021; Fouda et al., 2020; Ghovanloo \& Ruben, 2021; Zhang \& Bean, 2021). We previously characterized the full inhibitory effects of CBD on voltage-gated sodium channels (Nav) and deciphered the mechanism through which CBD inhibits Nav currents (Ghovanloo et al., 2018, 2021; Sait et al., 2020; Ghovanloo \& Ruben, 2021). We further found that an important component of this mechanism involves CBD altering membrane elasticity, which was measured using a gramicidin-based fluorescence assay (GFA) (Ingólfsson et al., 2010; Ghovanloo et al., 2021).

GFA is based on the gramicidin permeability to $\mathrm{Tl}^{+}$, a quencher of the water-soluble fluorophore 8-aminonaphthalene-1,3,6-trisulfonate (ANTS), encapsulated in large unilamellar vesicles (LUVs) doped with gramicidin. The rate of $\mathrm{Tl}^{+}$influx, measured as the rate of fluorescence quench, indicates the time-averaged number of gramicidin channels in the LUV membrane (Ingólfsson et al., 2010). Molecules that alter the thickness and elasticity of the LUV membrane also alter the lipid bilayer contribution to the free energy of dimerization and, thus, the free energy of dimerization (Rusinova et al., 2015). Our previous findings in LUVs suggested that CBD decreases gramicidin signals in that assay (Ghovanloo et al., 2021).

We sought to futher characterize CBD effects on gramaciding gramicidin currents using an electrophysiological HEK cell based assay.In the present study, we investigated the interactions between gramicidin and CBD over short exposures, using voltage-clamped human embryonic kidney (HEK-293) cells in the absence and presence of gramicidin. In this purely observational study, we report that in contrast to the GFA assay, CBD increases the gramicidin current in HEK cells. 


\section{RESULTS}

\section{CBD increases gramicidin signal in HEK cells in high extracellular sodium concentrations}

Gramicidin channels preferentially conduct cationic (e.g., $\mathrm{Na}^{+}$and $\mathrm{K}^{+}$) currents upon dimerization and pore formation (Harold \& Baarda, 1967; Hladky \& Haydon, 1970). We measured cationic currents through dimerized gramicidin channels using whole-cell voltage-clamp of untransfected HEK cells in the absence and presence of $26 \mu \mathrm{M}$ gramicidin applied to the extracellular side of the membrane. First, we measured gramicidin currents in standard high sodium $\left[\mathrm{Na}^{+}=140 \mathrm{mM}\right]$ extracellular solution using a ramp protocol. We clamped the cell membranes at $-80 \mathrm{mV}$, close to the $\mathrm{K}^{+}$equilibrium potential $\left(\mathrm{E}_{\mathrm{K}}{ }^{+}\right)$. Then, we hyperpolarized the cells to $-120 \mathrm{mV}$ and ramped the voltage to $+50 \mathrm{mV}$, which is close to $\mathrm{E}_{\mathrm{Na}}{ }^{+}$. We show average gramicidin current density from the ratio of current amplitude to the cell membrane capacitance $(\mathrm{pA} / \mathrm{pF})$ at $-120,-80,0$, and $+50 \mathrm{mV}$ (Figure 1A-D). Our results indicate that, at negative potentials, gramicidin conducts inward currents and, as the membrane potential becomes more positive, the current becomes outward with the reversal potential ( $\left.\mathrm{E}_{\mathrm{rev}}\right)$ being close to $0 \mathrm{mV}$, as would be predicted for a non-specific monovalent cationic channel. We also measured the effects of $1 \mu \mathrm{M}$ and $10 \mu \mathrm{M}$ CBD (Ghovanloo et al., 2018), and $10 \mu \mathrm{M}$ Triton X100 (TX100; as positive control (Lundbæk et al., 2004)) on gramicidin-HEK cells (Figure 1A-C). TX100 is a detergent, and has been shown to change membrane elasticity and hence to increase gramicidin current amplitude in equilibrium (Lundbæk et al., 2004). Interestingly, our findings indicate that TX100 reduced the cationic gramicidin currents across all potentials $(\mathrm{p}<0.05)$ (Figure 1C). CBD had the opposite effect to that of TX100, and slightly increased gramicidin currents at both $1 \mu \mathrm{M}(\mathrm{p}<0.05)$ and $10 \mu \mathrm{M}(\mathrm{p}>0.05)$. Interestingly, although the tendency for CBD to alter gramicidin currents was the same at both concentrations, CBD's effects were more variable at $10 \mu \mathrm{M}$ than at $1 \mu \mathrm{M}$; this variability resulted in lack of statistical significance at $10 \mu \mathrm{M}$ (Figure 1C). We speculate the variability at $10 \mu \mathrm{M}$ may be due to damage to the HEK cell membrane from both gramicidin and CBD over the timescales of voltage-clamp experiments.

\section{CBD increases gramicidin signal in HEK cells in low extracellular sodium concentrations}

The presence of a gramicidin dependent current indicates ion flux across the cell membrane. Gramacidin pores are analogous to puncturing cation-selective holes through the cell 
membrane. Gramicidin induced currents in the previous high $\left[\mathrm{Na}^{+}\right]$experiment resulted in a $E_{\text {rev }}$ close to $0 \mathrm{mV}$. This raises the possibility of a potential nonselective leak current component induced by gramicidin, but not carried by gramicidin, as a confounding variable. To ensure that we were recording gramicidin pore currents, we performed the same experiment with lower extracellular sodium $\left[\mathrm{Na}^{+}=1 \mathrm{mM}\right]$. This experiment resulted in the same overall trends of altered gramicidin currents densities as the high $\left[\mathrm{Na}^{+}\right]$experiment, for both $\mathrm{CBD}$ and TX100 (Figure 2AD). As expected, reducing $\left[\mathrm{Na}^{+}\right]$lowered the gramicidin $\mathrm{E}_{\text {rev }}$ to $\sim-80 \mathrm{mV}$ (close to $\mathrm{E}_{\mathrm{K}}^{+}$). These results confirm our results from the high $\mathrm{Na}^{+}$experiment and further suggest that, when both $\mathrm{Na}^{+}$ and $\mathrm{K}^{+}$are present at high concentrations, gramicidin permeability is not highly selective for $\mathrm{K}^{+}$ over $\mathrm{Na}^{+}$bringing the gramicidin $\mathrm{E}_{\text {rev }}$ to $\sim 0 \mathrm{mV}$. Overall, these results show that $\mathrm{CBD}$ increases gramicidin currents during short exposures and suggests, therefore, that CBD alters membrane elasticity or gramicidin channel conductance directly.

\section{DISCUSSION}

In our previous study, using a gramicidin-fluorescence assay (GFA), it was determined that CBD has the opposite effect to TX100 and that it decreases the rate of dimerized gramicidin channel formation, and hence a smaller gramicidin current. These findings indicate that CBD is bilateral modifying at the tested concentrations of 1-30 $\mu \mathrm{M}$ (Ghovanloo et al., 2021). In this study, by electrophysiologically measuring $\mathrm{K}+$ and $\mathrm{Na}+$ currents flowing through the gramicidin channel, the opposite result was observed. CBD increased gramicidin currents and decreased TX100 currents suggesting an alternate mechanism of gramacidin interaction with amphiphiles like CBD or TX100 than in the GFA assay.

Although the gramicidin structure does not indicate an obvious direct binding-site for CBD (Anon, n.d.), there is a chance of a direct CBD-gramicidin interaction taking place. Indeed, in almost every report of CBD activity on a given target, a response has been determined, including various ion channels and receptor proteins (Almeida \& Devi, 2020; Ghovanloo \& Ruben, 2021). Therefore, the opposite result that was observed that might suggest direct CBD and gramicidin interactions that in some way increase the probability of conducting pores in these conditions.

The molecular structure of CBD is composed of two oxygen atoms on both sides of a benzene ring, with the other two ends of the ring having a hydrocarbon tail on one end, and a hydrocarbon ring on the other. These features give the CBD molecule an overall shape that is 
loosely reminiscent of a phospholipid molecule. Phospholipids, in turn, are molecules that specialize in separating various cellular and sub-cellular environments, a function that is dependent on their amphiphilicity. In our previous paper, molecular dynamics (MD) simulations suggested that CBD molecules tend to localize below phospholipid headgroups, but above the tail-end region (Ghovanloo et al., 2021Thus, CBD molecules hovered around carbons $\sim 3-7$ of aliphatic chains, as per MD and verified by NMR (Ghovanloo et al., 2021). It is conceivable that interactions between CBD positioned in the leaflet of the HEK membrane may interact with gramicidin hemi channels to impact pore dimerization formation in a way that offset any membrane stiffness affects that inhibit gramicidin currents as we saw in previous GFA studies. In the GFA assay, gramicidin monomers are incubated for 24 hours with liposomes at $13{ }^{\circ} \mathrm{C}$ to reach equilibration, and then the effect of compound is investigated by measuring fluorescence quenching rates (Ingólfsson et al., 2010). In this study, we measure conventional macroscopic cationic currents in HEK cells using standard voltage-clamp, after the cells were extracellularly perfused with gramicidin monomers over the course of minutes at $27^{\circ} \mathrm{C}$. Therefore, the experimental setups between the two studies are fundamentally different, and likely investigate different phenomenon pertaining to gramicidin and CBD interactions.

Our goal in this study was to describe the effects of CBD on HEK cells externally treated with gramicidin. Our results suggest that there may be a direct interaction between CBD and gramicidin but the mechanism by which this potentiates gA currents in HEK cells remains unclear. Further studies will be required for instance, using MD simulations to examine potential for direct interactions.

\section{METHODS}

\section{Cell culture}

Suspension Human Embryonic Kidney (HEK-293) cells were used for automated patchclamp experiments. All cells were incubated at $37{ }^{\circ} \mathrm{C} / 5 \% \mathrm{CO}_{2}$. All cell culture reagents were purchased from ThermoFisher Scientific, Waltham, MA, unless otherwise noted.

\section{Patch-clamp}

Automated patch-clamp recording was performed on untransfected HEK cells. Currents were measured in the whole-cell configuration using a Qube-384 (Sophion A/S, Copenhagen, 
Denmark) automated voltage-clamp system. Intracellular solution contained (in mM): $120 \mathrm{KF}, 10$ $\mathrm{NaCl}, 2 \mathrm{MgCl}_{2}, 10 \mathrm{HEPES}$, adjusted to $\mathrm{pH} 7.2$ with $\mathrm{CsOH}$. The extracellular recording solution for the high sodium experiment contained (in $\mathrm{mM}$ ): $140 \mathrm{NaCl}, 3 \mathrm{KCl}, 1 \mathrm{MgCl}_{2}, 1.5 \mathrm{CaCl}_{2}, 10 \mathrm{HEPES}$, adjusted to pH7.4 with $\mathrm{NaOH}$. For the low sodium experiment the external solution sodium concentration was lowered to $1 \mathrm{mM}$ with $\mathrm{N}$-methyl-D-glucamine (NMDG) as $\mathrm{NaCl}$ replacement. Liquid junction potentials calculated to be $\sim 7 \mathrm{mV}$ were not adjusted for. Currents were low-passfiltered at $5 \mathrm{kHz}$ and recorded at $25 \mathrm{kHz}$ sampling frequency. Series resistance compensation was applied at 100\%. The measurements were obtained at room temperature which corresponds to 27 $\pm 2{ }^{\circ} \mathrm{C}$ at the recording chamber. Appropriate filters for cell membrane resistance (typically $>500$ $\mathrm{M} \Omega$ ) and series resistance ( $<10 \mathrm{M} \Omega)$ were used. Gramicidin was dissolved in 100\% DMSO, and the final concentration of $26 \mu \mathrm{M}$.

\section{Electrophysiological data analysis}

The analysis of raw patch-clamp recordings was performed using the Sophion Analyzer. Graphing and additional analysis was done using the Prism GraphPad (Version 9) software.

\section{Statistics}

A one-factor analysis of variance (ANOVA) or t-test were, when appropriate, were used to compare the mean responses. Post-hoc tests using the Tukey Kramer adjustment compared the mean responses between channel variants across conditions. A level of significance $\alpha=0.05$ was used in all overall post-hoc tests, and effects with p-values less than 0.05 were considered to be statistically significant. All values are reported as means \pm standard error of means (SEM) for $n$ recordings/samples.

\section{Funding and acknowledgements}

This work was supported by grants from Natural Science and Engineering Research Council of Canada and the Rare Disease Foundation to PCR and M-RG (CGS-D: 535333-2019 \& MSFSS: 546467-2019), a MITACS Accelerate fellowship in partnership with Xenon Pharma, Inc. to M-RG (IT10714).

M-RG assembled data, performed patch-clamp experiments, data analysis, figure making, wrote manuscript, data interpretation, and assisted to conceiving of experiments. SJG and PCR 
conceived the experiments and revised the manuscript critically. All co-authors edited the manuscript.

\section{Competing interests}

None. The authors declare that this research was conducted in the absence of competing interests. 


\section{REFERENCES}

Almeida DL \& Devi LA (2020). Diversity of molecular targets and signaling pathways for CBD. Pharmacol Res Perspect; DOI: 10.1002/prp2.682.

Andersen OS \& Koeppe RE (2007). Bilayer Thickness and Membrane Protein Function: An Energetic Perspective. Annu Rev Biophys Biomol Struct 36, 107-130.

Andersen OS, Koeppe RE \& Roux B (2005). Gramicidin channels. IEEE Trans Nanobioscience 4, 10-19.

Anon (n.d.). RCSB PDB - 1NRM: Gramicidin A in Dodecyl Phosphocholine Micelles (NMR). Available at: https://www.rcsb.org/structure/1NRM [Accessed April 26, 2020].

Devane WA, Dysarz FA, Johnson MR, Melvin LS \& Howlett AC (1988). Determination and characterization of a cannabinoid receptor in rat brain. Mol Pharmacol 34, 605-613.

Devinsky O, Cross JH, Laux L, Marsh E, Miller I, Nabbout R, Scheffer IE, Thiele EA, Wright S \& Cannabidiol in Dravet Syndrome Study Group (2017). Trial of Cannabidiol for DrugResistant Seizures in the Dravet Syndrome. N Engl J Med 376, 2011-2020.

Elsohly MA (2007). Marijuana and the Cannabinoids.

Finkelstein A (1974). Aqueous pores created in thin lipid membranes by the antibiotics nystatin, amphotericin B and gramicidin A. Implications for pores in plasma membranes. Drugs Transp Process B A Callingham, Ed London, UK MacMillan241-250.

Fouda MA, Ghovanloo MR \& Ruben PC (2020). Cannabidiol protects against high glucoseinduced oxidative stress and cytotoxicity in cardiac voltage-gated sodium channels. $\mathrm{Br} \mathrm{J}$ Pharmacol 177, 2932-2946.

Ghovanloo M-R, Choudhury K, Bandaru TS, Fouda MA, Rayani K, Rusinova R, Phaterpekar T, Nelkenbrecher K, Watkins AR, Poburko D, Thewalt J, Andersen OS, Delemotte L, Goodchild SJ \& Ruben PC (2021). Cannabidiol inhibits the skeletal muscle Nav1.4 by blocking its pore and by altering membrane elasticity. J Gen Physiol; DOI: 10.1085/jgp.202012701.

Ghovanloo M-R \& Ruben PC (2021). Cannabidiol and Sodium Channel Pharmacology: General Overview, Mechanism, and Clinical Implications. Neuroscientist 107385842110170.

Ghovanloo M-R, Shuart NG, Mezeyova J, Dean RA, Ruben PC \& Goodchild SJ (2018). Inhibitory effects of cannabidiol on voltage-dependent sodium currents. J Biol Chem 293, $16546-16558$.

Harold FM \& Baarda JR (1967). Gramicidin, Valinomycin, and Cation Permeability of Streptococcus faecalis. J Bacteriol94, 53.

Hladky SB \& Haydon DA (1970). Discreteness of conductance change in bimolecular lipid membranes in the presence of certain antibiotics. Nature 225, 451-453.

Hladky SB \& Haydon DA (1972). Ion transfer across lipid membranes in the presence of gramicidin A. I. Studies of the unit conductance channel. BBA - Biomembr 274, 294-312.

Iannotti FA, Pagano E, Moriello AS, Alvino FG, Sorrentino NC, D’Orsi L, Gazzerro E, Capasso R, De Leonibus E, De Petrocellis L \& Di Marzo V (2019). Effects of non-euphoric plant cannabinoids on muscle quality and performance of dystrophic mdx mice. Br J Pharmacol 176, 1568-1584.

Ingólfsson HI, Lea Sanford R, Kapoor R \& Andersen OS (2010). Gramicidin-based fluorescence assay; for determining small molecules potential for modifying lipid bilayer properties. $J$ Vis Exp; DOI: 10.3791/2131.

Kaplan JS, Stella N, Catterall WA \& Westenbroek RE (2017). Cannabidiol attenuates seizures 
and social deficits in a mouse model of Dravet syndrome. Proc Natl Acad Sci 114, 1122911234.

Kapoor R, Peyear TA, Koeppe RE \& Andersen OS (2019). Antidepressants are modifiers of lipid bilayer properties. J Gen Physiol 151, 342-356.

van Klingeren B \& ten Ham M (1976). Antibacterial activity of $\Delta 9$-tetrahydrocannabinol and cannabidiol. Antonie Van Leeuwenhoek42, 9-12.

Kosgodage US, Matewele P, Awamaria B, Kraev I, Warde P, Mastroianni G, Nunn A V., Guy GW, Bell JD, Inal JM \& Lange S (2019). Cannabidiol Is a Novel Modulator of Bacterial Membrane Vesicles. Front Cell Infect Microbiol; DOI: 10.3389/fcimb.2019.00324.

Lundbæk J (2005). CAPSAICIN REGULATES VOLTAGE-DEPENDENT SODIUM CHANNELSBY ALTERING LIPID BILAYER ELASTICITY. Mol Pharmacol 68, 680689.

Lundbæk JA, Birn P, Hansen AJ, Søgaard R, Nielsen C, Girshman J, Bruno MJ, Tape SE, Egebjerg J, Greathouse D V., Mattice GL, Koeppe RE \& Andersen OS (2004). Regulation of Sodium Channel Function by Bilayer Elasticity. J Gen Physiol 123, 599-621.

Lupica CR, Riegel AC \& Hoffman AF (2004). Marijuana and cannabinoid regulation of brain reward circuits. Br J Pharmacol 143, 227-234.

De Petrocellis L, Ligresti A, Moriello AS, Allarà M, Bisogno T, Petrosino S, Stott CG \& Di Marzo V (2011). Effects of cannabinoids and cannabinoid-enriched Cannabis extracts on TRP channels and endocannabinoid metabolic enzymes. Br J Pharmacol 163, 1479-1494.

Ross HR, Napier I \& Connor M (2008). Inhibition of recombinant human T-type calcium channels by $\Delta$ 9-tetrahydrocannabinol and cannabidiol. J Biol Chem 283, 16124-16134.

Rusinova R, Koeppe RE \& Andersen OS (2015). A general mechanism for drug promiscuity: Studies with amiodarone and other antiarrhythmics. J Gen Physiol 146, 463-475.

Sait LG, Sula A, Ghovanloo MR, Hollingworth D, Ruben PC \& Wallace BA (2020). Cannabidiol interactions with voltage-gated sodium channels. Elife 9, 1-17.

Zhang HXB \& Bean BP (2021). Cannabidiol inhibition of murine primary nociceptors: Tight binding to slow inactivated states of Nav1.8 channels. J Neurosci 41, 6371-6387. 


\section{Figure 1 - High sodium voltage-clamp, gramicidin (gA).}

(A) Shows the averaged cationic current densities of gramicidin in the presence/absence of $\mathrm{CBD}$ at 1 and $10 \mu \mathrm{M}$, and TX100 at $10 \mu \mathrm{M}$, on the left (in pA/pF, ECS: $-120 \mathrm{mV}=-13.6$ $\pm 6.3,-80 \mathrm{mV}=-10.1 \pm 5.0,0 \mathrm{mV}=5.2 \pm 0.5,+50 \mathrm{mV}=30.0 \pm 11.0, \mathrm{n}=36 ; \mathrm{gA}:-120 \mathrm{mV}$ $=-547.8 \pm 66.2,-80 \mathrm{mV}=-357 \pm 44,0 \mathrm{mV}=56.5 \pm 8.1,+50 \mathrm{mV}=441.1 \pm 60.5, \mathrm{n}=47$; $1 \mu \mathrm{M}$ CBD: $-120 \mathrm{mV}=-820.1 \pm 83.2,-80 \mathrm{mV}=-543 \pm 55.8,0 \mathrm{mV}=57.7 \pm 5.2,+50 \mathrm{mV}$ $=607.4 \pm 72.2, \mathrm{n}=56 ; 10 \mu \mathrm{M}$ CBD: $-120 \mathrm{mV}=-687.0 \pm 70.3,-80 \mathrm{mV}=-452.8 \pm 47.4,0$ $\mathrm{mV}=57.1 \pm 7.1,+50 \mathrm{mV}=649.2 \pm 118.8, \mathrm{n}=49 ; 10 \mu \mathrm{M}$ TX100: $-120 \mathrm{mV}=-330.8 \pm$ $43.8 \pm,-80 \mathrm{mV}=-216.5 \pm 30.4,0 \mathrm{mV}=37.3 \pm 4.0,+50 \mathrm{mV}=259.4 \pm 32.8, \mathrm{n}=48)$. The ramp voltage protocol is shown on the right. (B) Shows a cartoon diagram of how gramicidin monomers are thought to dimerize and form channels. (C) Shows quantification of the data shown in (A), stars indicate statistical significance. (D) Shows the associated current traces. 
Figure 2 - Low sodium voltage-clamp.

(A) Shows the averaged cationic current densities of gramicidin in the presence/absence of $\mathrm{CBD}$ at 1 and $10 \mu \mathrm{M}$, and TX100 at $10 \mu \mathrm{M}$ (in pA/pF, ECS: $-120 \mathrm{mV}=-4.1 \pm 0.9,-80 \mathrm{mV}$ $=-3.5 \pm 0.7,0 \mathrm{mV}=4.1 \pm 0.4,+50 \mathrm{mV}=18.8 \pm 1.7, \mathrm{n}=33 ; \mathrm{gA}:-120 \mathrm{mV}=-67.6 \pm 7.7$, $80 \mathrm{mV}=-23.0 \pm 2.9,0 \mathrm{mV}=189.8 \pm 20.6,+50 \mathrm{mV}=437.4 \pm 45.6, \mathrm{n}=45 ; 1 \mu \mathrm{M}$ CBD: $120 \mathrm{mV}=-90.9 \pm 9.0,-80 \mathrm{mV}=-30.6 \pm 2.9,0 \mathrm{mV}=261.7 \pm 28.7,+50 \mathrm{mV}=604.0 \pm 64.8$, $\mathrm{n}=55 ; 10 \mu \mathrm{M}$ CBD: $-120 \mathrm{mV}=-83.6 \pm 14.1,-80 \mathrm{mV}=-27.8 \pm 5.2,0 \mathrm{mV}=280.8 \pm 74.4$, $+50 \mathrm{mV}=624.9 \pm 149.6, \mathrm{n}=60 ; 10 \mu \mathrm{M}$ TX100: $-120 \mathrm{mV}=-26.3 \pm 3.5,-80 \mathrm{mV}=-9.7 \pm$ $1.3,0 \mathrm{mV}=74.2 \pm 11.3,+50 \mathrm{mV}=182.4 \pm 29.0, \mathrm{n}=50$ ). (B) Shows the ramp voltage protocol. (C) Shows quantification of the data shown in (A), stars indicate statistical significance. (D) Shows the associated current traces. 
bioRxiv preprint doi: https://doi.org/10.1101/2022.01.24.477627 t this version posted January 25, 2022. The copyright holder for this preprint (which was not certified by peer review) is the author/funder, who has granted bioRxiv a license to display the preprint in perpetuity. It is made available under aCC-BY-NC-ND 4.0 International license.

Figure 1
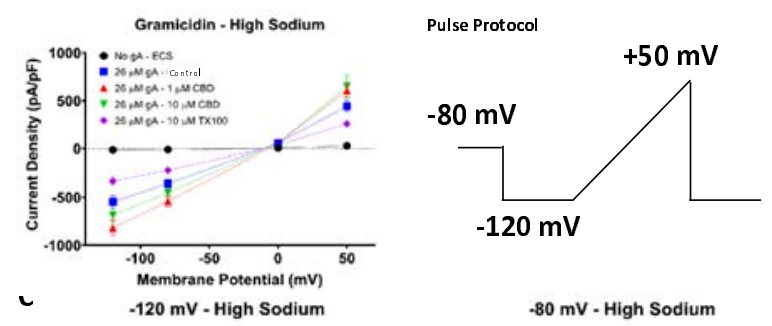

B
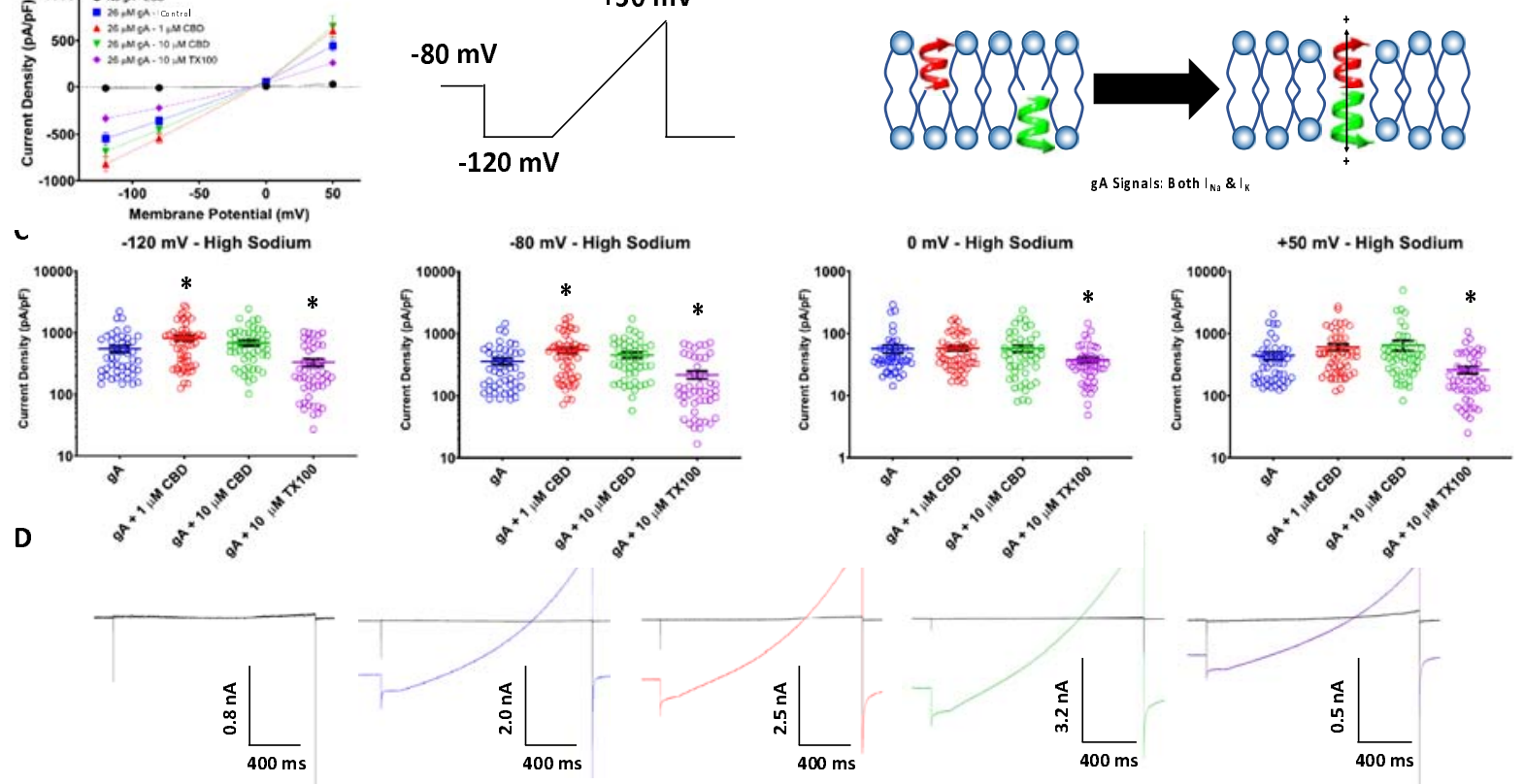

Figure 2

A

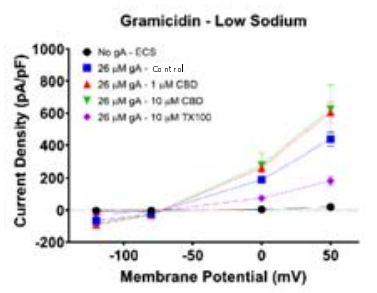

C
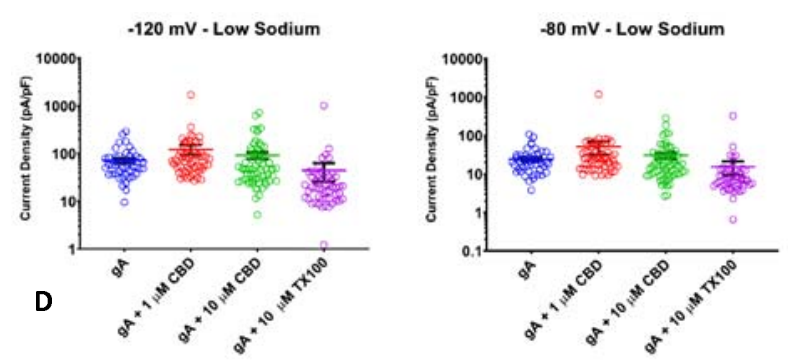

B

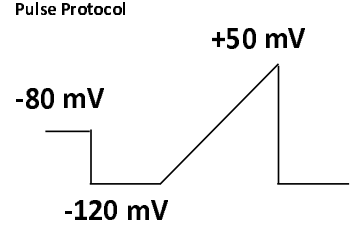

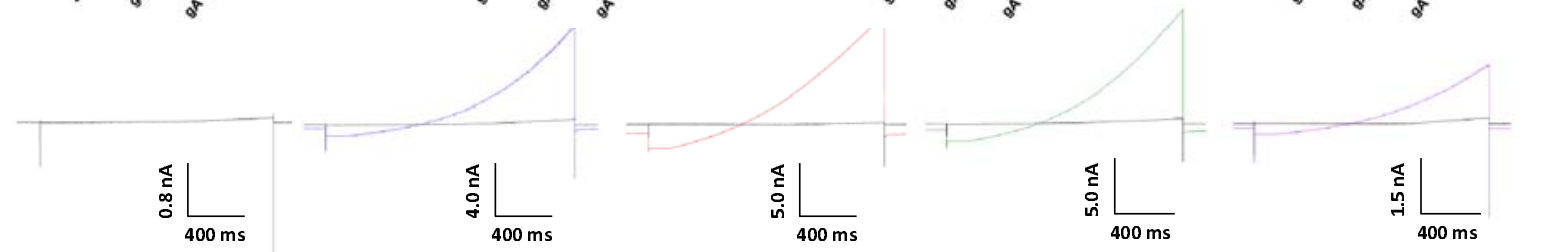

